\title{
Cardiac manifestations of COVID-19 infection: the role of echocardiography in patient management
}

\author{
Michael Dandel ${ }^{1}[$
}

Received: 11 August 2020 / Accepted: 13 August 2020 / Published online: 24 August 2020

(c) Springer-Verlag GmbH Germany, part of Springer Nature 2020

I read with interest the study by Zeng et al. [1]. Comparing the clinical and echocardiographic (ECHO) characteristics of patients treated in the intensive-care unit (ICU) with those of inpatients who did not necessitate intensive care, the study revealed valuable data which provide important insights into the highly complex and still barely known issue of cardiac manifestations related to COVID-19 infection.

Although COVID-19 infection is mainly known for causing severe respiratory pathology, it can also result in substantial, even life-threatening cardiac manifestations and there is increasing evidence that cardiovascular involvement plays often a central role in disease progression and patient outcomes [2].

The pathophysiology underlying cardiac involvement during COVID-19 infection is likely to be more often multi-factorial (e.g., direct viral toxicity, endothelial damage inflammation and thrombotic events) [2]. It has been observed that almost one third of the hospitalized non-critically ill COVID-19 infected patients can exhibit pulmonary hypertension $(\mathrm{PH})$ and/or right ventricular (RV) dysfunction, and that patients who necessitate cardiorespiratory therapy in an ICU can develop isolated RV failure (RVF), more often associated with increased pulmonary vascular resistance (PVR) related to high-pressure mechanical ventilation or derived from pulmonary embolism and/or small pulmonary vessel in situ thrombosis [2]. Zeng et al. also found that $29 \%$ of COVID-19 infected patients treated in the ICU had a pulmonary arterial systolic pressure (PAPS) $>40 \mathrm{mmHg}$ [1]. In other studies, among the critically ill Covid-19-confirmed patients, almost 50\% revealed thrombotic events (including distal pulmonary microvascular occlusions) and about $22 \%$ experienced myocardial ischemia and/or myocarditis [2-4]. This emphasizes the necessity to adapt the management of

Michael Dandel

mdandel@aol.com

1 German Centre for Heart and Circulatory Research (DZHK) Partner Site Berlin, Berlin, Germany such patients to the individual particularities of the major pathomechanisms involved in the development and persistence of their cardiorespiratory failure. All these data also suggest that it can be of vital importance to differentiate between pneumonia with and without relevant pulmonary vessel thrombosis, between biventricular failure and isolated RVF, as well as between primary and secondary RV dysfunction. This in turn can facilitate the selection of the most appropriate management (e.g. RV protective ventilation, anticoagulation, pulmonary vasodilation, extracorporeal membrane oxygenation, etc.) which can be decisive for patient survival. For such differentiations it is necessary to monitor cardiac function which is most easily achievable by bedside transthoracic ECHO (TTE), which is very suitable for this propose, largely available and safe for the patient.

In the study by Zeng et al., special attention deserves the observation that among the 57 inpatients who received TTE (ICU group 31 patients, non-ICU group 26 patients) there were no differences in the median LVEF values between the ICU and non-ICU group (63.00 [59.00-66.00] and 63.50 [60.00-67.00], respectively) although in the ICU group 5 patients $(16 \%)$ had a reduced LVEF $(<55 \%)$ [1]. Also in the ICU group, only 2 patients (6\%) had an enlarged LV (enddiastolic diameter $>55 \mathrm{~mm}$ ) and only 1 patient had a left atrial (LA) diameter $>35 \mathrm{~mm}$. This might suggest that myocardial systolic function is possibly less frequently directly affected by COVID-19 infection and may support the generally recommended restrictive use of cardiac imaging in order to prevent the spread of the virus. However, from a hemodynamic point of view, the right-sided heart and pulmonary circulation appeared more relevantly involved in the COVID-19 induced cardio-pulmonary alterations and raises many questions which cannot be reliably answered without the help of TTE. Figure $2 \mathrm{f}$ presented by Zang et al. underlines this aspect in a convincing manner [1]. In that figure, the pressure difference of $59 \mathrm{mmHg}$ between the RV and right atrium $\left(\Delta \mathrm{P}_{\mathrm{RV}-\mathrm{RA}}\right)$ during $\mathrm{RV}$ systole in the presence of relevant TR indicates a PAPS of 70-75 $\mathrm{mmHg}$, which can 
induce RVF by afterload mismatch even in patients without alteration of myocardial contractility.

Given the increasing evidence for the high risk of RV overloading in COVID-19 infected patients it becomes increasingly difficult to follow the initial recommendation to avoid "unnecessary" cardiac imaging examinations (including TTE) in order to reduce transmission of the virus and protect healthcare professionals, because without any ECHO examination it is hardly possible to establish whether monitoring of cardiac anatomy and function is indeed unnecessary in a particular patient. In fact, with the ongoing COVID-19 pandemic, bedside point-of-care ultrasound (POCUS) or limited TTE plays increasingly critical roles for driving decisions for COVID-19-positive patients [2].

To increase the efficiency of POCUS simultaneously with reduction of TTE duration, it is important to focus on essential ECHO parameters. At the same time, due to the limited possibilities to use all ECHO tools otherwise available, one must take into consideration also the major limitations of certain basic parameters in reliable assessment quantification of cardiac morphological and functional alterations. Standardized diameter measurements of the heart chambers in different two-dimensional (2D) ECHO long- and shortaxis views, as well as calculation of ventricular short/long $(S / L)$ axis-ratios allow reliable and reproducible detection and quantification of alterations in their size and geometry. Interatrial septum position, which is dependent on the interatrial pressure relation and interventricular septum position during systole and diastole, can also provide important indirect information on intracavitary pressure changes.

During assessment of ventricular function, special attention should be paid to the RV. Compared to the LV, ECHO assessment of RV systolic function is more challenging and the interpretation of RV systolic parameters is more demanding due to their higher sensitivity to afterload changes and the substantial contribution of LV contraction to the RV pump function [5]. The 2D-ECHO-derived ejection fraction (EF) for assessment of RV function is not any more recommended because the complex RV shape with poor definition of its endocardial surface and the limited imaging windows make the EF measurements less reliable. For routine assessment of RV systolic function more useful appeared to be the easy measurable $\mathrm{RV}$ fractional area-change $\left(\mathrm{FAC}_{\mathrm{RV}}\right)$ and lateral tricuspid annulus peak systolic excursion (TAPSE), which require no geometric assumptions and can provide similar information to RVEF [5]. $\mathrm{FAC}_{\mathrm{RV}}$ reflects both longitudinal and radial myofiber contribution to the overall RV function but measurements can be hampered by poor endocardial definition (especially in systole) and its reliability is limited by not including the contribution of the RV outflow tract (RVOT) to ejection [5]. TAPSE measurements are angle dependent and influenced by LV function and overall heart motion [5]. Another problem is the overvaluation of its diagnostic value in assuming that displacement of a single segment can represent the function of the entire RV although TAPSE does not take into account the contribution of the IVS and/or the RVOT to RV performance [5]. Due to their load dependency, $\mathrm{FAC}_{\mathrm{RV}}$ and TAPSE may change without changes in myocardial contractility (e.g., can decrease with increasing PVR even if RV contractility remains unaltered) [5]. Tricuspid regurgitation (TR) can induce misleading $\mathrm{FAC}_{\mathrm{RV}}$ and TAPSE changes [5]. By facilitating the RV free wall transversal (inward) and longitudinal motion, which will increase $\mathrm{FAC}_{\mathrm{RV}}$ and TAPSE (correspondingly to the increased blood volume leaving the RV in systole), TR can lead to an overestimation of RV systolic function [5]. Tissue Doppler imaging (TDI) derived lateral tricuspid annulus peak systolic velocity (TAPS') is also useful for evaluation of RV function. The limitations of TAPS' are similar to those of TAPSE and its reliability is also impaired by the "tethering phenomenon" (intrinsic limitation of TDI) by which the motion of the normal myocardium surrounding the diseased area can result in falsely normal tissue velocities of the diseased region [5]. Another problem is that the sample volume is fixed and does not enable tracking of the region of interest as it translates with the cardiac cycle and respiration.

A fundamental question which arises particularly in patients with infection-related relevant cardiorespiratory disturbances is whether RV dysfunction is induced by pressure overloading or by impaired contractility, or even by both. RV contractile abilities appeared most accurately reflected by the relationship between RV pressure load and alteration of RV size and geometry. A simple approach to assess this relationship can be the calculation of the " $\mathrm{RV}$ load-adaptation index" $\left(\mathrm{LAI}_{\mathrm{RV}}\right)$, which is a composite ECHO-derived variable based on the relationship between RV load and RV dilation, also taking the RA pressure into account (i.e. ratio between the systolic mean $\Delta \mathrm{P}_{\mathrm{RV}-\mathrm{RA}}$ and $\mathrm{RV}$ end-diastolic volume per long-axis length) [5]. Because a RV with good adaptation to high afterload can develop higher systolic pressure (without RV dilation or increase in RA pressure) in response to high resistance in the pulmonary circulation, the higher the $\mathrm{LAI}_{\mathrm{RV}}$, the better will be the adaptation of its contractile function. Using the TR velocity-time integral ( $\left(\mathrm{VTI}_{\mathrm{TR}}\right)$ instead of $\Delta \mathrm{P}_{\mathrm{RV}-\mathrm{RA}}$ and the easily measurable $\mathrm{RV}$ end-diastolic area $\left(\mathrm{A}_{\mathrm{ED}}\right)$ in the apical 4-chamber view instead of the RV end-diastolic volume (EDV), the $\mathrm{LAI}_{\mathrm{RV}}$ defined as:

$$
\mathrm{LAI}_{\mathrm{RV}}=\frac{\mathrm{VTI}_{\mathrm{TR}}(\mathrm{cm}) \cdot L_{\mathrm{ED}}(\mathrm{cm})}{A_{\mathrm{ED}}\left(\mathrm{cm}^{2}\right)} \text { becomes an easy calculable }
$$

dimensionless index [5]. Thus, a small RV area relative to long-axis length (size and geometry unaltered) in a patient with high $\mathrm{VTI}_{\mathrm{TR}}$ (i.e. high RV systolic pressure and relatively low RA pressure) yield a high $\mathrm{LAI}_{\mathrm{RV}}$ which indicates good adaptation to load (suggesting also a good RV 
contractility) and the potential of the RV to improve its performance after reduction of loading conditions. A large $A_{E D}$ relative to long-axis length (spherical dilation) despite a relatively low $\mathrm{VTI}_{\mathrm{TR}}$ yields a low $\mathrm{LAI}_{\mathrm{RV}}$ indicating poor adaptation to load (suggesting also a reduced myocardial contractility). $\mathrm{LAI}_{\mathrm{RV}}$ values $\geq 18$ indicate good $\mathrm{RV}$ adaptability, whereas $\mathrm{LAI}_{\mathrm{RV}}$ values $<15$ indicate low adaptability to load which will be insufficient to prevent RV dysfunction even at normal PVR [5]. A good RV adaptability to high afterload does not exclude a reduced RV pump function because this will inevitably occur in case of afterload mismatch [5]. Thus, the $\mathrm{LAI}_{\mathrm{RV}}$ allows distinguishing between pressure overloading-induced and impaired contractilityinduced RV dysfunction, as well as between patients with similar FAC $_{\mathrm{RV}}$, TAPSE or TAPS', but different RV myocardial inotropy [5]. In PAH, the $\mathrm{LAI}_{\mathrm{RV}}$ can reach values of 25 , even in patients with RVF suggesting that in the vast majority of PAH patients the cause of RVF is the excessive pressure overload and not a relevantly impaired RV contractility [5]. This explains the reverse remodeling with normalization of RV function in the overwhelming majority of patients with pre-capillary PH-induced severe RV dysfunction after they underwent lung transplantation [5].

Because handheld laptop-based equipment is sufficient for the bedside acquisition of the entire above mentioned essential ECHO data it appears absolutely advisable to further promote the use of this time and cost-effective diagnostic tool in the management of symptomatic COVID-19 infected patients.

\section{Compliance with ethical standards}

Conflict of interest The authors declare that they have no competing interests.

\section{References}

1. Zeng J-H, Wu W-B, Qu J-X, et al. Cardiac manifestations of COVID-19 in Shenzhen. China Infect. 2020;28:1-10. https://doi. org/10.1007/s15010-020-01473-w.

2. Gupta A, Madhavan MV, Sehgal K, et al. Extrapulmonary manifestations of COVID-19. Nat Med. 2020;26(7):1017-32.

3. Clerkin KJ, Fried JA, Raikhelkar J, et al. COVID-19 and cardiovascular disease. Circulation. 2020;141(20):1648-55.

4. Price LC, McCabe C, Garfield B, Wort SJ. Thrombosis and COVID-19 pneumonia: the clot thickens! Eur Respir J. 2020;56(1):2001608. https://doi.org/10.1183/13993003.01608 -2020 .

5. Dandel M, Hetzer R. Evaluation of the right ventricle by echocardiography: particularities and major challenges. Expert Rev Cardiovasc Ther. 2018;16(4):259-75. 